\title{
Modeling Multi-Order Adaptive Processes by Self-Modeling Networks
}

\author{
Jan TREUR ${ }^{1}$ \\ Social AI Group, Vrije Universiteit Amsterdam, De Boelelaan 1111, $1081 \mathrm{HV}$ \\ Amsterdam, the Netherlands
}

\begin{abstract}
A self-modeling network for some base network is a network extension that represents part of the base network structure by a self-model in terms of added network nodes and connections for them. By iterating this construction, multi-order network adaptation is easily obtained. A dedicated software environment for selfmodeling networks that has been developed supports the modeling and simulation processes. This will be illustrated for a number of adaptation principles from a number of application domains.
\end{abstract}

Keywords. Adaptive network, self-modeling network, multi-order adaptive

\section{Introduction}

A self-modeling network is a network that represents part of its own network structure by a self-model in terms of dedicated network nodes and connections for them. A network structure can be described by network characteristics for connectivity for connections between nodes, aggregation for combining multiple incoming impacts on a node, and timing for the speed of node state dynamics; e.g., [1, 2, 3]. Any base network can be extended to a self-modeling network for it, by adding a self-model for part of the base network's structure. In this case, the added self-model consists of a number of added nodes representing specific characteristics of the base network structure, such as connection weights and excitability thresholds, plus connections for these added selfmodel nodes. For the approach considered here, in general nodes in a network are assumed to have activation levels that can change over time due to impact from other nodes from which they have incoming connections. If in particular the nodes from a selfmodel representing some of the network characteristics of a base network are dynamic, these base network characteristics become adaptive, thus an adaptive base network is obtained, in the sense that adaptation of the base network is modeled by the dynamics within the self-modeling network extending the base network.

Moreover, multi-order network adaptation can be obtained by iterating this selfmodeling construction. If multi-order self-models are included in a self-modeling network, any included self-model (of some order) can have its own (next-order) selfmodel within the overall network where the latter self-model represents some of the network characteristics of the former self-model. For example, this allows to control the dynamics of self-models, so that self-controlled adaptive networks are obtained.

\footnotetext{
${ }^{1}$ Corresponding Author: Jan Treur, Social AI Group, Vrije Universiteit Amsterdam, De Boelelaan 1111, 1081HV Amsterdam, the Netherlands; Email: j.treur@vu.nl.
} 
A dedicated software environment for self-modeling networks that has been developed supports these modeling and simulation processes; see [3], Ch. 9. In this paper, for a number of adaptation principles from different application domains, it will be illustrated how they can be modeled by proper pre-specified self-models that can be used as building blocks to extend any base network to make it adaptive.

In the paper, first in Section 2 the modeling approach from [3] based on selfmodeling networks is briefly described. In Section 3 nine different adaptation principles from the Cognitive Neuroscience and Social Science literature are described. Next, in Section 4, for the adaptation principles described in Section 3 it is shown in more detail how they can be modeled by self-models. Section 5 is a discussion.

\section{Networks Using Self-Models: Self-Modeling Networks}

In this section, the network-oriented modelling approach used from [3] is introduced. Following [3,4], a temporal-causal network model is characterized by (here $X$ and $Y$ denote nodes of the network, also called states):

- Connectivity characteristics Connections from a state $X$ to a state $Y$ and their weights $\omega_{X, Y}$

Aggregation characteristics For any state $Y$, some combination function $\mathbf{c}_{Y}(.$. (usually with some parameters) defines the aggregation that is applied to the impacts $\omega_{X, Y} X(t)$ on $Y$ from its incoming connections from states $X$

- Timing characteristics Each state $Y$ has a speed factor $\eta_{Y}$ defining how fast it changes for given impact.

The following difference (or differential) equations that are used for simulation purposes and also for analysis of temporal-causal networks incorporate these network characteristics $\omega_{X, Y}, \mathbf{c}_{Y}(.),. \eta_{Y}$ in a standard numerical format:

$$
Y(t+\Delta t)=Y(t)+\eta_{Y}\left[\mathbf{c}_{Y}\left(\omega_{X_{1}, Y} X_{1}(t), \cdots, \omega_{X_{k}, Y} X_{k}(t)\right)-Y(t)\right] \Delta t
$$

for any state $Y$ and where $X_{1}$ to $X_{k}$ are the states from which $Y$ gets its incoming connections. Here the overall combination function $\mathbf{c}_{Y}(.$.$) for state Y$ is the weighted average of available basic combination functions $\mathbf{c}_{j}(.$.$) by specified weights \gamma_{j, Y}$ (and parameters $\boldsymbol{\pi}_{1, j, Y}, \boldsymbol{\pi}_{2, j, Y}$ of $\left.\mathbf{c}_{j}(.).\right)$ for $Y$ :

$$
\mathbf{c}_{Y}\left(V_{1}, \ldots, V_{k}\right)=\frac{\gamma_{1, Y} \mathrm{c}_{1}\left(V_{1}, \ldots, V_{k}\right)+\ldots+\gamma_{m, Y} c_{m}\left(V_{1}, \ldots, V_{k}\right)}{\gamma_{1, Y}+\ldots+\gamma_{m, Y}}
$$

Such Eq. (1) and (2) are hidden in the dedicated software environment; see [3], Ch 9. Within this software environment, currently around 40 useful basic combination functions are included in a combination function library; see Table 1 for some of them. The above concepts enable to design network models and their dynamics in a declarative manner, based on mathematically defined functions and relations.

Table 1. Examples of basic combination functions from the library.

\begin{tabular}{llll}
\hline & Notation & Formula & Parameters \\
\hline Euclidean & $\operatorname{eucl}_{n, \lambda}\left(V_{1}, \ldots, V_{k}\right)$ & $\sqrt[n]{\frac{V_{1}^{n}+\cdots+V_{k}^{n}}{\lambda}}$ & $\begin{array}{l}\text { Order } \boldsymbol{n}>0 \\
\text { Scaling factor } \lambda>0\end{array}$ \\
$\begin{array}{l}\text { Advanced } \\
\text { logistic sum }\end{array}$ & $\operatorname{alogistic}_{\sigma, \tau}\left(V_{1}, \ldots, V_{k}\right)$ & {$\left[\frac{1}{1+\mathrm{e}^{-\boldsymbol{\sigma}\left(V_{1}+\cdots+V_{k}-\tau\right)}}-\frac{1}{\left.\left.1+\mathrm{e}^{\sigma \tau}\right)\right]\left(1+\mathrm{e}^{-\boldsymbol{\sigma} \tau}\right)}\right.$} & $\begin{array}{l}\text { Steepness } \boldsymbol{\sigma}>0 \\
\text { Excitability threshold } \tau\end{array}$ \\
$\begin{array}{l}\text { Scaled } \\
\text { maximum }\end{array}$ & $\operatorname{smax}_{\lambda}\left(V_{1}, \ldots, V_{k}\right)$ & $\max \left(V_{1}, \ldots, V_{k}\right) / \lambda$ & Scaling factor $\lambda>0$ \\
$\begin{array}{l}\text { Scaled } \\
\text { minimum }\end{array}$ & $\operatorname{smin}_{\lambda}\left(V_{1}, \ldots, V_{k}\right)$ & $\min \left(V_{1}, \ldots, V_{k}\right) / \lambda$ & Scaling factor $\lambda>0$ \\
\hline
\end{tabular}


Note that there is a crucial distinction for network models between network characteristics and network states. Network states have values (their activation levels) and are explicit representations that may be accessible for network states by connections to and from them and can be handled or manipulated in that way. They can be considered to provide an informational view on the network; usually the states are assumed to have a certain informational content. In contrast, network characteristics (such as connection weights and excitability thresholds) have values (their strengths) and determine (e.g., cognitive) processes and behavior in an implicit, automatic manner. They can be considered to provide an embodiment view on the network. In principle, these characteristics by themselves are not directly accessible nor observable for network states; in principle you can make connections between states but you cannot make connections between network characteristics or between states and characteristics.

As indicated above, 'network characteristics' and 'network states' are two distinct concepts for a network. Self-modeling is a way to relate these distinct concepts to each other in an interesting and useful way. A self-model is making the network characteristics (such as connection weights and excitability thresholds) explicit in the form of adding states (called self-model states) for these characteristics and also connections for these additional states. Thus, the network gets an internal self-model of part of its network structure: it explicitly represents information about its own network structure. In this way, by iteration different self-modeling levels can be created where network characteristics from one level relate to network states at a next level. Thus, an arbitrary number of selfmodeling levels can be modeled, covering second-order or higher-order effects.

More specifically, adding a self-model for a temporal-causal base network is done in the way that for some of the states $Y$ of the base network and some of the network structure characteristics for connectivity, aggregation and timing (i.e., some from $\boldsymbol{\omega}_{X, Y}$, $\boldsymbol{\gamma}_{j, Y}, \boldsymbol{\pi}_{i, j, Y}, \boldsymbol{\eta}_{Y}$ ), additional network states $\mathbf{W}_{X, Y}, \mathbf{C}_{j, Y}, \mathbf{P}_{i, j, Y}, \mathbf{H}_{Y}$ (self-model states or reification states) are introduced and connected to other states:

\section{a) Connectivity self-model}

- Self-model states $\mathbf{W}_{X, Y}$ are added representing connectivity characteristics, in particular connection weights $\boldsymbol{\omega}_{X, Y}$

b) Aggregation self-model

- Self-model states $\mathbf{C}_{j, Y}$ are added representing aggregation characteristics, in particular combination function weights $\gamma_{j, Y}$

- Self-model states $\mathbf{P}_{i, j, Y}$ are added representing aggregation characteristics, in particular combination function parameters $\boldsymbol{\pi}_{i, j, Y}$

c) Timing self-model

- Self-model states $\mathbf{H}_{Y}$ are added representing timing characteristics, in particular speed factors $\eta_{Y}$

The notations $\mathbf{W}_{X, Y}, \mathbf{C}_{j, Y}, \mathbf{P}_{i, j, Y}, \mathbf{H}_{Y}$ for the self-model states indicate the referencing relation with respect to the characteristics $\boldsymbol{\omega}_{X, Y}, \boldsymbol{\gamma}_{j, Y}, \boldsymbol{\pi}_{i, j, Y}, \boldsymbol{\eta}_{Y}$ : here $\mathbf{W}$ refers to $\boldsymbol{\omega}, \mathbf{C}$ refers to $\gamma, \mathbf{P}$ refers to $\boldsymbol{\pi}$, and $\mathbf{H}$ refers to $\eta$, respectively. For the processing, these self-model states define the dynamics of any state $Y$ in a canonical manner according to Eq. (1) and (2) whereby the values of $\boldsymbol{\omega}_{X, Y}, \gamma_{j, Y}, \boldsymbol{\pi}_{i, j, Y}, \boldsymbol{\eta}_{Y}$ are replaced by the state values of $\mathbf{W}_{X, Y}, \mathbf{C}_{j, Y}$, $\mathbf{P}_{i, j, Y}, \mathbf{H}_{Y}$ at time $t$, respectively.

Note that concerning the terminology used, only the states that represent some network characteristics are called self-model states. The states to which these self-model states are connected still belong to the self-model (e.g., as depicted in Figure 1 and 
further) but they can either be other self-model states or other states that are not selfmodel states, such as the states $X$ and $Y$. An example of an aggregation self-model state $\mathbf{P}_{i, j, Y}$ for a combination function parameter $\boldsymbol{\pi}_{i, j, Y}$ is for the excitability threshold $\boldsymbol{\tau}_{Y}$ of state $Y$, which is the second parameter of a logistic sum combination function; then $\mathbf{P}_{i, j, Y}$ is usually indicated by $\mathbf{T}_{Y}$, where $\mathbf{T}$ refers to $\tau$. The network constructed by the addition of a self-model to a base network is called a self-modeling network or a reified network for this base network. This constructed network is also a temporal-causal network model itself, as has been shown in [3], Ch. 10; for this reason, this construction can easily be applied iteratively to obtain multiple levels or orders of self-models, in which case the resulting network is called a multi-order or higher-order self-modeling network or reified network.

\section{Adaptation Principles from Different Domains}

In this section, a number of adaptation principles of different orders are described as can be found in the literature on Cognitive Neuroscience and Social Sciences.

\subsection{First-order Adaptation Principles}

First-order adaptation principles for some base network address adaptation of some of the base network's characteristics concerning its connectivity, aggregation of multiple connections and timing of node state dynamics. Much research has focused in particular on learning of connectivity characteristics based on adaptive connections, but also other characteristics can be made adaptive, as will be discussed.

\subsubsection{The Hebbian Learning Adaptation Principle}

As a first example, for mental or neural networks, the Hebbian learning adaptation principle [5] can be formulated by:

'When an axon of cell A is near enough to excite B and repeatedly or persistently takes part in firing it, some growth process or metabolic change takes place in one or both cells such that A's efficiency, as one of the cells firing B, is increased.' [5], p. 62

This is sometimes simplified (neglecting the phrase 'one of the cells firing B') to:

'What fires together, wires together' [6, 7]

This can easily be modeled by using a connectivity self-model based on self-model states $\mathbf{W}_{X, Y}$ representing connection weights $\omega_{X, Y}$.

\subsubsection{The Bonding by Homophily Adaptation Principle}

An example of the use of a network's self-model for the social domain is the bonding by homophily adaptation principle

'Birds of a feather flock together'

This expresses how being 'birds of a feather' or 'being alike' strengthens the connection between two persons [8-13]. Similar to the Hebbian learning case, this can be modeled by a social network's connectivity self-model based on self-model states $\mathbf{W}_{X, Y}$ representing connection weights $\omega_{X, Y}$. 


\subsubsection{The More Becomes More Adaptation Principle}

Another first-order adaptation principle for social networks is the 'more becomes more' principle expressing that more popular people attract more connections:

'Persons with more connections attract more connections' [4], p. 311

In a wider context this more becomes more principle relates to what sometimes is called 'the rich get richer' $[14,15]$, 'cumulative advantage' [16], 'the Matthew effect' [17] or 'preferential attachment' [18]. Similar to the Hebbian learning and bonding by homophily cases, this can be modeled by a social network's connectivity self-model based on self-model states $\mathbf{W}_{X, Y}$ representing connection weights $\boldsymbol{\omega}_{X, Y}$.

\subsubsection{The Interaction Connects Adaptation Principle}

The idea behind the Interaction Connects adaptation principle from Social Science is that 'The more interaction you have with somebody, the stronger you will become connected' (6)

See, for example, [19-23]. Similar to the Hebbian learning and bonding by homophily cases, this can be modeled by a social network's connectivity self-model based on selfmodel states $\mathbf{W}_{X, Y}$ representing connection weights $\boldsymbol{\omega}_{X, Y}$.

\subsubsection{The Enhanced Excitability Adaptation Principle}

Although connectivity adaptation has some popularity in the literature, also other characteristics can be made adaptive. Instead of a connectivity self-model to model adaptive connection weights, also an aggregation self-model can be used, for example, to model intrinsic neuronal excitability, as described in [24]:

'Long-lasting modifications in intrinsic excitability are manifested in changes

in the neuron's response to a given extrinsic current (generated by synaptic

activity or applied via the recording electrode).' [24], p. 30

This form of adaptation can be modeled by an aggregation self-model based on selfmodel states $\mathbf{T}_{Y}$ for adaptive excitability thresholds. For example, this type of self-model has been used to model adaptation (desensitization) to spicy food; see [25].

\subsection{Second-Order Adaptation Principles}

The examples of adaptation principles in Section 3.1 refer to forms of plasticity, which can be described by a first-order adaptive network that is modelled using a dynamic firstorder self-model for connectivity or aggregation characteristics of the base network, in particular for the connection weights and/or the excitability thresholds used in aggregation. Whether or not and to which extent such plasticity as described above actually takes place is controlled by a form of metaplasticity; e.g. [26-31].

\subsubsection{The Exposure Accelerates Adaptation Speed Adaptation Principle}

For example, in [29] the following compact quote is found indicating that due to stimulus exposure, the adaptation speed will increase:

'Adaptation accelerates with increasing stimulus exposure' [29], p. 2.

This indeed refers to a form of metaplasticity, which can be described by a second-order adaptive network that is modeled using a dynamic second-order timing self-model, for timing characteristics of a first-order self-model for the first-order adaptation, based on self-model states $\mathbf{H}_{\mathbf{w}_{X, Y}}$ for adaptive learning speed. 


\subsubsection{The Exposure Modulates Persistence Adaptation Principle}

A similar perspective can be applied to obtain a principle for modulation of persistence.

'Stimulus exposure modulates persistence of adaptation'

Depending on further context factors, this can be applied in different ways. Reduced persistence can be used in order to be able to get rid of earlier learnt connections that do not apply. However, enhanced persistence can be used to keep what has been learnt. This also refers to a form of metaplasticity, which can be described by a second-order adaptive network that is modeled using a dynamic second-order aggregation self-model, for persistence characteristics of a first-order self-model for the first-order adaptation, based on self-model states $\mathbf{M}_{\mathbf{W}_{X, Y}}$ for an adaptive persistence factor.

\subsubsection{The Plasticity Versus Stability adaptation principle}

In a similar direction [31] it is more generally discussed how it depends on the circumstances when the extent of plasticity is or should be high and when it is or should be low in favor of stability:

'The Plasticity Versus Stability Conundrum' [31], p. 773.

This principle relates to the previous two and can use these second-order self-models.

\subsubsection{The Stress Blocks Adaptation Principle}

Yet another principle that is indicated in the literature refers to the effect of high stress levels on the extent of plasticity:

'High stress levels slow down or block adaptation'

See, for example, the following quote from [27], where such slowing down or blocking of adaptation is called negative metaplasticity:

'Numerous electrophysiological studies have shown that 'negative' metaplasticity develops

in brain areas such as the hippocampus and its related structures (e.g., the lateral septum and

the nucleus accumbens) following stress.' [27], p. 631

This can be described by a second-order adaptive network modeled using a dynamic second-order timing self-model, for timing characteristics of a first-order self-model for the first-order adaptation, based on self-model states $\mathbf{H}_{X, Y}$ for adaptive learning speed.

The first- and second-order adaptation principles such as the one summarized in (3) to (11) above have been formalized in the form of self-models used in first- and secondorder adaptive network models that have been designed, as discussed in Section 4.

\section{Using Self-Models to Formalize Adaptation Principles}

In this section, it will be shown how the modeling approach for self-modeling network models described in Section 2 can be used to model the adaptation principles of different orders discussed in Section 3. In particular the connectivity and aggregation characteristics of the addressed self-models are discussed. Timing characteristics for these self-models are just values (speed factors for each of the states) that will usually be set depending on a specific application. When self-models are changing over time in a proper manner, this offers a useful method to model adaptive networks based on any adaptation principles. This does not only apply to first-order adaptive networks, but also to higher-order adaptive networks, by using higher-order self-models. 


\subsection{First-Order Self-Models for First-Order Adaptation Principles}

First the adaptation principle for Hebbian Learning will be addressed, as described in Section 3.1.1. To incorporate the 'firing together' part, for the self-model's connectivity characteristics, upward causal connections to connectivity self-model state $\mathbf{W}_{X, Y}$ from $X$ and $Y$ are used to formulate a Hebbian learning adaptation principle; see Figure 1. The upward connections have weight 1 here. Also a connection from $\mathbf{W}_{X, Y}$ to itself with weight 1 is used; in pictures they are usually left out.

So, the connectivity characteristics of the self-model here consist of the three nodes $\mathbf{W}_{X, Y}, X$, and $Y$, together with the two incoming upward connections (the blue arrows) from $X$ and $Y$ to $\mathbf{W}_{X, Y}$, one outgoing connection from $\mathbf{W}_{X, Y}$ to $Y$ (the pink downward arrow), and the leveled connection (black arrow) from $X$ to $Y$. Note that as mentioned in the last paragraph of Section 3.2, only the states that represent a network characteristic are called self-model states, in this case $\mathbf{W}_{X, Y}$. In connectivity pictures such as Figure 1 and further, the self-model states are the states with an outgoing (pink) downward connection. Some other states to which they are connected such as in this case $X$ and $Y$ are still part of the self-model, but will not be called self-model states; they do not have an outgoing downward connection. The downward connection takes care that the value of $\mathbf{W}_{X, Y}$ is actually used for the connection weight of the connection from $X$ to $Y$. For the aggregation characteristics of the self-model, one of the options for a learning rule is defined by the combination function $\operatorname{hebb}_{\mu}\left(V_{1}, V_{2}, W\right)$ from Table 2 , where $V_{1}, V_{2}$ refer to the activation levels of the connected states $X$ to $Y$, and $W$ to the value of $\mathbf{W}_{X, Y}$ representing the connection weight. For more options of Hebbian learning combination functions and further mathematical analysis of them, see, for example [3], Ch. 14.

Table 2 Combination functions for self-models modeling the first- and second-order adaptation principles. The first five rows cover the first-order adaptation principles from Section 3.1 and the last four rows the secondorder adaptation principles from Section 3.2.

\begin{tabular}{|c|c|c|c|}
\hline $\begin{array}{l}\text { Adaptation principle an } \\
\text { self-model state }\end{array}$ & & $\begin{array}{l}\text { Combination function } \\
\text { options }\end{array}$ & $\begin{array}{l}\text { Variables and } \\
\text { Parameters }\end{array}$ \\
\hline $\begin{array}{l}\text { Hebbian Learning } \\
\mathbf{W}_{X, Y}\end{array}$ & 3.1 .1 & $\begin{array}{l}\mathbf{h e b b}_{\boldsymbol{\mu}}\left(V_{1}, V_{2}, W\right)= \\
V_{1} V_{2}(1-W)+\boldsymbol{\mu} W\end{array}$ & $\begin{array}{l}V_{1}, V_{2} \text { activation levels of connected states } \\
W \text { activation level of self-model state for } \\
\text { connection weight } \\
\mu \text { persistence factor }\end{array}$ \\
\hline $\begin{array}{l}\text { Bonding by Homophily } \\
\mathbf{W}_{X, Y}\end{array}$ & 3.1 .2 & $\begin{array}{l}\operatorname{slhomo}_{\alpha, \tau}\left(V_{1}, V_{2}, W\right)= \\
W+\alpha W(1-W)\left(\tau-\left|V_{1}-V_{2}\right|\right)\end{array}$ & $\begin{array}{l}V_{1}, V_{2} \text { activation levels of connected persons } \\
W \text { connection weight } \\
\alpha \text { modulation factor } \\
\tau \text { tipping point }\end{array}$ \\
\hline $\begin{array}{l}\text { More Becomes More } \\
\mathbf{W}_{X, Y}\end{array}$ & 3.1 .3 & $\begin{array}{l}\operatorname{eucl}_{n, \lambda}\left(W_{1}, \ldots, W_{k}\right) \\
\operatorname{alogistic}_{\sigma, \tau}\left(W_{1}, \ldots, W_{k}\right)\end{array}$ & $\begin{array}{l}W_{1}, \ldots, W_{k} \text { activation levels of self-model } \\
\text { states for connection weights of persons } \\
\text { connected to B }\end{array}$ \\
\hline $\begin{array}{l}\text { Interaction Connects } \\
\mathbf{W}_{X, Y}\end{array}$ & 3.1 .4 & $\begin{array}{l}\operatorname{eucl}_{n, \lambda}\left(V_{1}, \ldots, V_{k}\right) \\
\operatorname{alogistic}_{\sigma, \tau}\left(V_{1}, \ldots, V_{k}\right)\end{array}$ & $\begin{array}{l}V_{1}, \ldots, V_{k} \text { impacts from interaction states for } \\
\text { the connected person }\end{array}$ \\
\hline $\begin{array}{l}\text { Enhanced Excitability } \\
\mathbf{T}_{Y}\end{array}$ & 3.1 .5 & $\begin{array}{l}\operatorname{eucl}_{n, \lambda}\left(V_{1}, \ldots, V_{k}\right) \\
\operatorname{alogistic}_{\sigma, \tau}\left(V_{1}, \ldots, V_{k}\right)\end{array}$ & $V_{1}, \ldots, V_{k}$ impacts from base states \\
\hline $\begin{array}{l}\text { Exposure Accelerates } \\
\text { Adaptation Speed } \mathbf{H}_{\mathbf{W}_{X Y}}\end{array}$ & 3.2 .1 & $\begin{array}{l}\operatorname{eucl}_{n, \lambda}\left(V_{1}, \ldots, V_{k}\right) \\
\operatorname{alogistic}_{\sigma, \tau}\left(V_{1}, \ldots, V_{k}\right)\end{array}$ & $\begin{array}{l}V_{1}, \ldots, V_{k} \text { impacts from base states and first- } \\
\text { order self-model states }\end{array}$ \\
\hline $\begin{array}{l}\text { Exposure Modulates } \\
\text { Persistence } \mathbf{M}_{\mathbf{W}_{X, Y}}\end{array}$ & 3.2 .2 & $\begin{array}{l}\operatorname{eucl}_{n, \lambda}\left(V_{1}, \ldots, V_{k}\right) \\
\operatorname{alogistic}_{\sigma, \tau}\left(V_{1}, \ldots, V_{k}\right)\end{array}$ & $\begin{array}{l}V_{1}, \ldots, V_{k} \text { impacts from base states and first- } \\
\text { order self-model states }\end{array}$ \\
\hline $\begin{array}{l}\text { Plasticity Versus } \\
\text { Stability } \mathbf{H}_{\mathbf{W}_{X Y Y}}, \mathbf{M}_{\mathbf{W}_{X, Y}}\end{array}$ & 3.2 .3 & $\begin{array}{l}\operatorname{eucl}_{n, \lambda}\left(V_{1}, \ldots, V_{k}\right) \\
\operatorname{alogistic}_{\sigma, \tau}\left(V_{1}, \ldots, V_{k}\right)\end{array}$ & $\begin{array}{l}V_{1}, \ldots, V_{k} \text { impacts from base states and first- } \\
\text { order self-model states }\end{array}$ \\
\hline $\begin{array}{l}\text { Stress Blocks } \\
\text { Adaptation } \mathbf{H}_{\mathbf{W}_{X, Y}}\end{array}$ & 3.2 .4 & $\begin{array}{l}\operatorname{eucl}_{n, \lambda}\left(V_{1}, \ldots, V_{k}\right) \\
\operatorname{alogistic}_{\sigma, \tau}\left(V_{1}, \ldots, V_{k}\right)\end{array}$ & $\begin{array}{l}V_{1}, \ldots, V_{k} \text { impacts from base states for stress } \\
\text { level and first-order self-model states }\end{array}$ \\
\hline
\end{tabular}


Next, the adaptation principle for Bonding by homophily will be addressed, as described in Section 3.1.2. It happens that for this connectivity self-model exactly the same connectivity characteristics apply as for Hebbian learning, as depicted in Figure 1.

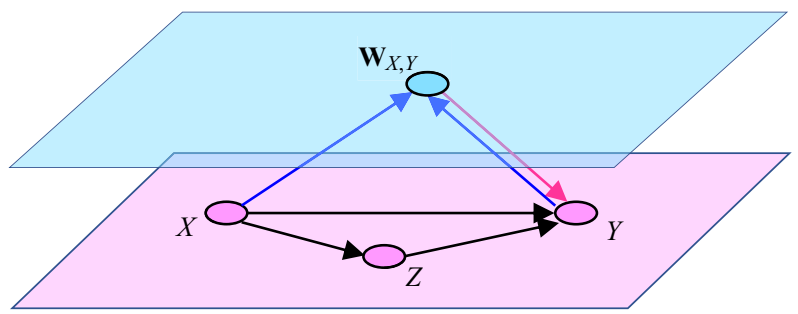

Figure 1. Connectivity characteristics of the self-model for the Hebbian Learning adaptation principle for Mental Networks or the Bonding by Homophily adaptation principle for Social Networks

For aggregation characteristics of this self-model, an option for an adaptation rule is defined by the combination function slhomo $\boldsymbol{s}_{\alpha, \tau}\left(V_{1}, V_{2}, W\right)$ from Table 2, where $V_{1}, V_{2}$ refer to the activation levels (for example, for some opinion) of the connected persons and $W$ to the value of $\mathbf{W}_{X, Y}$ representing the connection weight. For more options and further mathematical analysis, see, for example [3], Ch. 13, or [13].

The More Becomes More adaptation principle as described in Section 3.1.3 has connectivity characteristics as shown in Figure 2. Here, the connectivity self-model states for different connections affect each other, as a connection of a person $X_{3}$ to a given person $Y$ depends on the existence and strengths of connections from other persons $X_{i}$ to the same person $Y$; see the black leveled arrows in the upper plane.

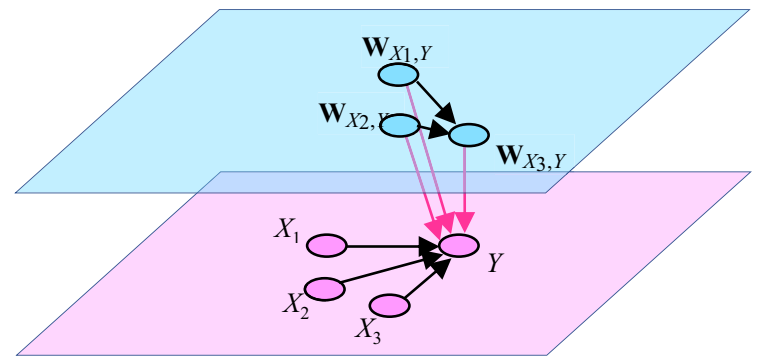

Figure 2. Connectivity characteristics of a self-model for the More Becomes More adaptation principle for person $X_{3}$ with respect to person $Y$

So, in this case the connectivity characteristics of the self-model are the nodes $\mathbf{W}_{X_{1}, Y}$, $\mathbf{W}_{X_{2}, Y}, \mathbf{W}_{X_{3}, Y}$, and $Y$, together with leveled connections (black arrows) from each $\mathbf{W}_{X_{j}, Y}$ to $\mathbf{W}_{X 3, Y}$ and downward connections (pink arrows) from each $\mathbf{W}_{X_{j}, Y}$ to $Y$. Again, these (pink) downward connections takes care that the value of $\mathbf{W}_{X_{j}, Y}$ is actually used for the connection weight of the connection from $X_{j}$ to $Y$. For the aggregation characteristics of this self-model, some form of aggregation of the weights of these other connections represented by the $\mathbf{W}_{X j, Y}$ can be used, such as by using a Euclidean or logistic sum combination function; see Table 2. For example, in [32] a logistic sum function was used, and in [33] a scaled sum (with scaling factor the number of existing connections for $Y$ resulting in an average weight), which is a first-order Euclidean combination function.

For the Interaction Connects adaptation principle described in Section 3.1.4, the connectivity self-model states for the connection weights are affected by certain states 
$I_{X_{j}, Y}$ representing the strength of (actual) interaction. Therefore, the connectivity characteristics of a self-model for this adaptation principle are as shown in Figure 3, with (blue) upward connections from interaction states $I_{X_{i}, Y}$ to the self-model states $\mathbf{W}_{X_{i}, Y}$ and (pink) downward connections from $\mathbf{W}_{X_{i}, Y}$ to $Y$. Note that there also multiple interaction states can be used for one connection, for example, for different interaction channels. The aggregation characteristics of the self-model states $\mathbf{W}_{X_{i}, Y}$ can be specified, for example, by a Euclidean or logistic sum function, as shown in Table 2.

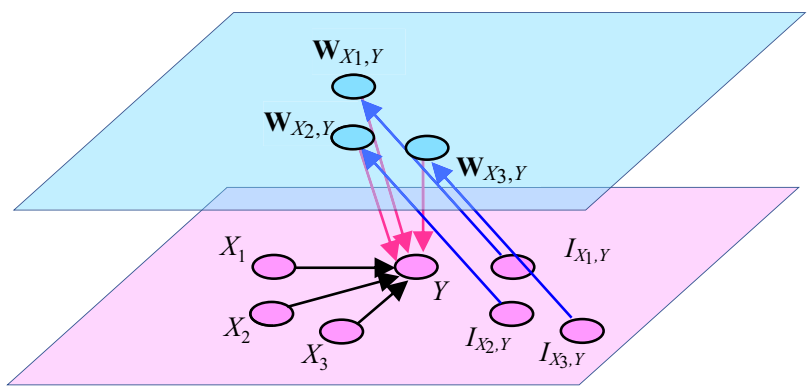

Figure 3. Connectivity characteristics of a self-model for the Interaction Connects adaptation principle for persons $X_{3}, X_{2}$ and $X_{3}$ with respect to person $Y$.

For the Enhanced Excitability adaptation principle described in Section 3.1.5, an aggregation self-model with connectivity characteristics depicted in Figure 4 can be used.

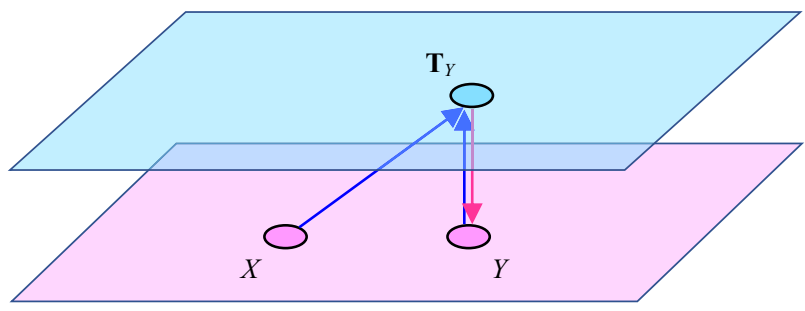

Figure 4. Connectivity characteristics of a self-model for the Enhanced Excitability adaptation principle for persons $X_{3}, X_{2}$ and $X_{3}$ with respect to person $Y$.

In this case state $Y$ is assumed to use a logistic sum combination function, which has an excitability threshold parameter $\tau$ (or any other function with such a parameter). Here this excitability threshold is represented by aggregation self-model state $\mathbf{T}_{Y}$ which is affected by exposure from activation of the involved states. Note that to enhance excitability, the value of self-model state $\mathbf{T}_{Y}$ representing the excitability threshold has to decrease. Therefore, these upward connections need to get negative connection weights, whereas a positive connection weight from $\mathbf{T}_{Y}$ itself can be used. In this case, the (pink) downward connection from $\mathbf{T}_{Y}$ to $Y$ takes care that the value of $\mathbf{T}_{Y}$ is actually used for the threshold value of the logistic sum function of $Y$. Also a connection from a related connectivity self-model state $\mathbf{W}_{X, Y}$ to $\mathbf{T}_{Y}$ with positive connection weight might be added in this self-model to obtain some balancing effect. For the aggregation characteristics, for example, a Euclidean (with odd order $\boldsymbol{n}$ to keep the negative impacts negative) or logistic sum function can be used for $\mathbf{T}_{Y}$, as shown in Table 2. 


\subsection{Second-Order Self-Models for Second-Order Adaptation Principles}

The first second-order adaptation principle discussed is the Exposure Accelerates Adaptation Speed principle described in Section 3.2.1. This is modeled by a second-order timing self-model. As it is a second-order adaptation principle for some first-order adaptation principle, for the sake of clarity it is described here with respect to the firstorder adaptation principle for Hebbian Learning; although it might be applied to other first-order adaptation principle as well, but then it will have a similar structure to what is shown here. The connectivity characteristics of this timing self-model are shown in Figure 5; they consist of the states $\mathbf{H W}_{X, Y}, \mathbf{W}_{X, Y}, X$, and $Y$, together with the (positive, blue) upward connections from the two base states $X$ and $Y$ to the self-model state $\mathbf{H w}_{X, Y}$ expressing the part of the principle referring to 'exposure', the (negative, blue) upward connection from $\mathbf{W}_{X, Y}$ to the self-model state $\mathbf{H}_{\mathbf{W}_{X, Y}}$, and the downward (pink) connection from $\mathbf{H W}_{X, Y}$ to $\mathbf{W}_{X, Y}$ that takes care that the value of $\mathbf{H W}_{X, Y}$ is actually used as speed factor for $\mathbf{W}_{X, Y}$. By the upward connections, stronger activation of the base states $X$ and $Y$ will lead to an increased value of $\mathbf{H W}_{X, Y}$, as indicated by the part of the principle referring to 'accelerates'. The (negative) upward connection from the considered state $\mathbf{W}_{X, Y}$ to $\mathbf{H W}_{X, Y}$ can be used for (counter)balancing. For the aggregation characteristics, for example a Euclidean (with odd order $\boldsymbol{n}$ to keep the negative impacts negative) or logistic sum function can be used for $\mathbf{H W}_{X, Y}$, as shown in Table 2 .

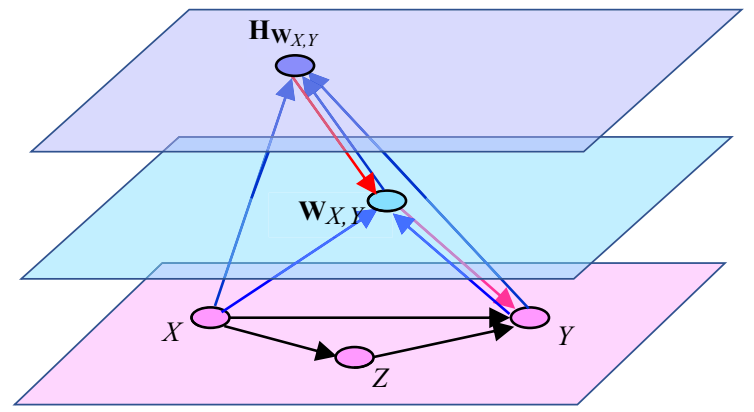

Figure 5. Connectivity of a second-order self-model for the Exposure Accelerates Adaptation Speed adaptation principle with a first-order self-model for Hebbian learning.

Next, the second-order Exposure Modulates Persistence adaptation principle (for the first-order Hebbian Learning principle) described in Section 3.2.2 is addressed, based on second-order aggregation self-model state $\mathbf{M W}_{X, Y}$ representing persistence of the firstorder adaptation. For the connectivity characteristics of this self-model, see Figure 6.

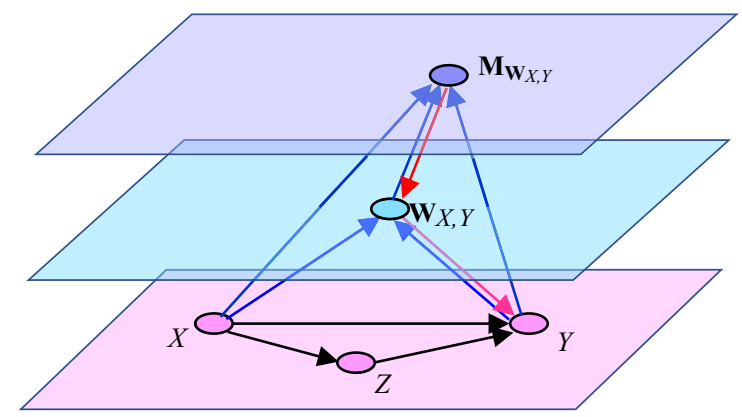

Figure. 6. Connectivity of a second-order self-model for the Exposure Modulates Persistence adaptation principle with a first-order self-model for Hebbian learning 
The upward connections from base states $X$ and $Y$ to $\mathbf{M W}_{X, Y}$ may suppress the persistence (when they are negative). This paves the road to get rid of the learnt effects from the past in case they are no longer applicable. The positive upward connection from first-order state $\mathbf{W}_{X, Y}$ to $\mathbf{H W}_{X, Y}$ can be used for counterbalancing. However, the upward connections from base states $X$ and $Y$ to $\mathbf{M}_{X, Y}$ can also be made positive in which case they increase persistence during a learning process to keep the learnt effect well. This also illustrates the Plasticity Versus Stability Conundrum adaptation principle described in Section 3.2.3. The (pink) downward connection from $\mathbf{M W}_{X, Y}$ to $\mathbf{W}_{X, Y}$ takes care that the value of $\mathbf{W}_{X, Y}$ is actually used for the connection weight of the connection from $X$ to $Y$. For the aggregation characteristics, for example a Euclidean (with odd order $\boldsymbol{n}$ ) or logistic sum function can be used for $\mathbf{M} \mathbf{W}_{X, Y}$, as shown in Table 2.

Finally, a second-order self-model for the Stress Blocks Adaptation principle described in Section 3.2.4 can be obtained in a similar way as the one for Exposure Accelerates Adaptation Speed principle (see connectivity in Figure 5) but this time with connectivity characteristics based on a negative upward connection from a base state representing the stress level, which brings the timing characteristic self-model state $\mathbf{H W}_{X, Y}$, to low values or even 0. For the aggregation characteristics, again for example a Euclidean or logistic sum function can be used for $\mathbf{H W}_{X, Y}$; see Table 2.

\section{Discussion}

In this paper the use of self-modeling networks to model adaptive biological, mental and social processes of any order of adaptation was addressed. Following the networkoriented modeling approach described in [3], it was shown how self-models for networks provide useful pre-specified building blocks to design complex multi-order adaptive network models in the form of self-modeling networks. This was illustrated for a number of adaptation principles from different application domains. A dedicated software environment for self-modeling networks that has been developed supports the modeling and simulation: https://www.researchgate.net/project/Network-Oriented-Modeling-Software.

As an illustration, in [3], Ch. 4, four of the adaptation principles known from the literature and specified in Section 4 were applied to obtain a network model involving both plasticity and metaplasticity. In particular, two first-order adaptation principles (for Hebbian Learning and for Enhanced Excitability) and two second-order adaptation principles (for Exposure Accelerates Adaptation Speed and for Exposure Modulates Persistence) are covered in this network model.

\section{References}

[1] Treur J. Multilevel network reification: representing higher order adaptivity in a network. In: Aiello L, Cherifi C, Cherifi H, Lambiotte R, Lió P, Rocha L. editors. Proc. of the $7^{\text {th }}$ Int. Conf. on Complex Networks and their Applications, ComplexNetworks'18, vol. 1. Studies in Computational Intelligence, vol. 812, Springer Nature, 2018, p. 635-51.

[2] Treur J. Modeling higher-order adaptivity of a network by multilevel network reification. Network Science 2020;8: S110-44.

[3] Treur J. Network-oriented modeling for adaptive networks: designing higher-order adaptive biological, mental, and social network models. Cham, Switzerland: Springer Nature Publishing; 2020. 412 p. 
[4] Treur J. Network-Oriented Modeling: Addressing Complexity of Cognitive, Affective and Social Interactions. Cham, Switzerland: Springer Publishers; 2016. 499 p.

[5] Hebb DO. The organization of behavior: A neuropsychological theory. New York: John Wiley and Sons; 1949. $335 \mathrm{p}$.

[6] Shatz CJ. The developing brain. Sci. Am. 1992; 267:60-67. (10.1038/scientificamerican0992-60)

[7] Keysers C, Gazzola V. Hebbian learning and predictive mirror neurons for actions, sensations and emotions. Philos Trans R Soc Lond B Biol Sci 2014;369: 20130175.

[8] Pearson M, Steglich C, Snijders T. Homophily and assimilation among sport-active adolescent substance users. Connections 2006;27(1):47-63.

[9] McPherson M, Smith-Lovin L, Cook JM. Birds of a feather: homophily in social networks. Annu. Rev. Sociol. 2001; 27:415-44.

[10] Levy DA, Nail PR. Contagion: A theoretical and empirical review and reconceptualization. Genetic, social, and general psychology monographs 1993;119(2):233-284.

[11] Holme P, Newman MEJ. Nonequilibrium phase transition in the coevolution of networks and opinions Phys. Rev. E 2006;74(5):056108.

[12] Sharpanskykh A, Treur J. Modelling and analysis of social contagion in dynamic networks. Neurocomputing 2014; 146:140-50.

[13] Treur J. Mathematical analysis of the emergence of communities based on coevolution of social contagion and bonding by homophily. Applied Network Science 2019;4: article 1.

[14] Simon HA. On a class of skew distribution functions Biometrika 1955; 42: 425-40.

[15] Bornholdt S, Ebel H. World wide webscaling exponent from Simon's 1955 model Phys. Rev. E 2001;64: article 035104.

[16] Price DJ. de S. A general theory of bibliometric and other cumulative advantage processes J. Amer. Soc. Inform. Sci. 1976; 27: 292-306

[17] Merton RK. The Matthew effect in science. Science 1968;159: 56-63.

[18] Barabási AL, Albert R. Emergence of scaling in random networks. Science 1999; 286: 509-512.

[19] Hove MJ, Risen JL. It's all in the timing: interpersonal synchrony increases affiliation. Soc. Cognit. 2009; 27: 949-60. (doi:10.1521/soco. 2009.27.6.949)

[20] Pearce E, Launay J, Dunbar RIM. (). The Ice-breaker Effect: singing together mediates fast social bonding. Royal Society Open Science 2015;2: article 150221 http://dx.doi.org/10.1098/ rsos.150221.

[21] Weinstein D, Launay J, Pearce, E, Dunbar RIM, Stewart L. Singing and social bonding: Changes in connectivity and pain threshold as a function of group size. Evolution \& Human Behaviour 2016;37(2):152-58. doi: 10.1016/j.evolhumbehav.2015.10.002

[22] Gilbert E, Karahalios K. Predicting tie strength with social media. Proceedings of the SIGCHI Conference on Human Factors in Computing Systems CHI'09, 2009, p. 211-20.

[23] Morris MR, Teevan J, Panovich K. What do people ask their social networks, and why? a survey study of status message Q\&A behavior. CHI 2010. 2010.

[24] Chandra N, Barkai E. A non-synaptic mechanism of complex learning: modulation of intrinsic neuronal excitability. Neurobiology of Learning and Memory 2018; 154: 30-36.

[25] Choy M, El Fassi S, Treur J. An adaptive network model for pain and pleasure through spicy food and its desensitization. Cognitive Systems Research 2020: in press

[26] Abraham WC, Bear MF. Metaplasticity: the plasticity of synaptic plasticity. Trends in Neuroscience 1996;19(4):126-130.

[27] Garcia R. Stress, metaplasticity, and antidepressants. Current Molecular Medicine 2002; 2: 629-38.

[28] Magerl W, Hansen N, Treede RD, Klein T. The human pain system exhibits higher-order plasticity (metaplasticity). Neurobiology of Learning and Memory 2018; 154:112-20.

[29] Robinson BL, Harper NS, McAlpine D. Meta-adaptation in the auditory midbrain under cortical influence. Nat. Commun. 2016; 7: article 13442.

[30] Sehgal M, Song C, Ehlers VL, Moyer Jr JR. Learning to learn - intrinsic plasticity as a metaplasticity mechanism for memory formation. Neurobiology of Learning and Memory 2013; 105: 186-99.

[31] Sjöström PJ, Rancz EA, Roth A, Hausser M. Dendritic excitability and synaptic plasticity. Physiol Rev 2008; 88: 769-840.

[32] Beukel S van den, Goos SH, Treur J. An adaptive temporal-causal network model for social networks based on the homophily and more-becomes-more principle. Neurocomputing 2019; 338: 361-71

[33] Blankendaal R, Parinussa S, Treur J. A temporal-causal modelling approach to integrated contagion and network change in social networks. Proceedings of the Twenty-second European Conference on Artificial Intelligence, ECAI'16, 2016, p. 1388-96 